\title{
Infrared exponents of Yang-Mills theory
}

\section{Christian S. Fischer*}

IPPP, Durham

E-mail: christian.fischer\&oturham.ac.uk

In this talk I summarise recent results on the infrared exponents of $\mathrm{SU}\left(N_{c}\right)$-Yang-Mills theory. I discuss a self-consistent power law solution for the Dyson-Schwinger equations for general 1PI-Greens functions in the infrared. The corresponding running coupling has a fixed point at zero momentum, which turns out to be universal and gauge invariant within a class of transverse gauges. When calculated on a torus the infrared exponents of the ghost and gluon propagators differ from the corresponding continuum solutions. They agree, however, well with results from lattice calculations.

XXIIIrd International Symposium on Lattice Field Theory

25-30 July 2005

Trinity College, Dublin, Ireland

${ }^{*}$ Speaker. 
The infrared behaviour of the Green's functions of $\mathrm{SU}\left(N_{c}\right)$-Yang-Mills theory is related to confinement in several interesting ways. One example is the Kugo-Ojima confinement criterion, which is satisfied in Landau gauge if the dressing function of the ghost propagator is singular and the gluon propagator is finite or vanishes in the infrared [1,2]. A second example is provided by the colour Coulomb potential, which can be determined from the instantaneous part of the time-time component of the Coulomb gauge gluon propagator [3]. In this talk I summarise recent analytical results on the infrared exponents of the Green's functions of SU( $\left.N_{c}\right)$-Yang-Mills theory in Landau gauge [4] and a class of gauges that interpolate between Landau and Coulomb gauge [5]. Furthermore, I discuss the relation of these solutions with corresponding results from DysonSchwinger equations (DSEs) and lattice simulations on a compact manifold [6].

\section{Infrared exponents in the continuum: Landau gauge}

The basic idea to determine the infrared behaviour of one-particle-irreducible (1PI) Green's functions is to investigate their Dyson-Schwinger equations order by order in a skeleton expansion (i.e. a loop expansion using full propagators and vertices). It turns out that these Green's functions can only be infrared singular, if all external scales go to zero. Thus to determine the degree of possible singularities it is sufficient to investigate the DSEs in the presence of only one external scale $p^{2} \ll \Lambda_{Q C D}^{2}$. In collaboration with R. Alkofer and F. Llanes-Estrada we showed by induction that the skeleton expansion is stable in the sense that higher orders generate terms that are equally or less singular than those of lower orders. Thus one can determine the infrared behaviour of these functions from their DSEs approximated to lowest order in the skeleton expansion. We found a self-consistent solution of the tower of DSEs for 1PI-Green's functions, given by [4]

$$
\Gamma^{n, m}\left(p^{2}\right) \sim\left(p^{2}\right)^{(n-m) \kappa} .
$$

Here $\Gamma^{n, m}\left(p^{2}\right)$ denotes the infrared leading dressing function of the 1PI-Green's function with $2 n$ external ghost legs and $m$ external gluon legs. The exponent $\kappa$ is known to be positive [7], independent of any truncation scheme of the DSEs.

A special instance of the solution (1.1) are the inverse ghost and gluon dressing functions $\Gamma^{1,0}\left(p^{2}\right)=G^{-1}\left(p^{2}\right)$ and $\Gamma^{0,2}\left(p^{2}\right)=Z^{-1}\left(p^{2}\right)$, which are related to the ghost and gluon propagators via

$$
D^{G}\left(p^{2}\right)=-\frac{G\left(p^{2}\right)}{p^{2}}, \quad D_{\mu \nu}\left(p^{2}\right)=\left(\delta_{\mu v}-\frac{p_{\mu} p_{v}}{p^{2}}\right) \frac{Z\left(p^{2}\right)}{p^{2}} .
$$

The corresponding power laws in the infrared are

$$
G\left(p^{2}\right) \sim\left(p^{2}\right)^{-\kappa}, \quad Z\left(p^{2}\right) \sim\left(p^{2}\right)^{2 \kappa} .
$$

In this notation the Kugo-Ojima criterion translates to the condition $\kappa \geq 0.5$. For a bare ghostgluon vertex in the infrared, justified by lattice calculations [8] and also in the DSE-approach [9], one obtains $\kappa=(93-\sqrt{1201}) / 98 \approx 0.595[10,11]$, which satisfies the criterion. This specific value of $\kappa$ is found to vary only slightly for a large class of possible dressings of the ghost-gluonvertex [11]. Similar values have been determined from exact renormalisation group equations [12].

An interesting consequence of the solution (1.1) is the qualitative universality of the running coupling in the infrared. Renormalisation group invariant couplings can be defined from either of 
the primitively divergent vertices of Yang-Mills-theory, i.e. from the ghost-gluon vertex $(g h-g l)$, the three-gluon vertex $(3 g)$ or the four-gluon vertex $(4 g)$ via

$$
\begin{array}{rlrl}
\alpha^{g h-g l}\left(p^{2}\right) & =\frac{g^{2}}{4 \pi} G^{2}\left(p^{2}\right) Z\left(p^{2}\right) & \stackrel{p^{2} \rightarrow 0}{\sim} \text { const } / N_{c}, \\
\alpha^{3 g}\left(p^{2}\right) & =\frac{g^{2}}{4 \pi}\left[\Gamma^{0,3}\left(p^{2}\right)\right]^{2} Z^{3}\left(p^{2}\right) \stackrel{p^{2} \rightarrow 0}{\sim} \text { const } / N_{c}, \\
\alpha^{4 g}\left(p^{2}\right) & =\frac{g^{2}}{4 \pi}\left[\Gamma^{0,4}\left(p^{2}\right)\right]^{2} Z^{4}\left(p^{2}\right) \stackrel{p^{2} \rightarrow 0}{\sim} \text { const } / N_{c} .
\end{array}
$$

Using the DSE-solution (1.1) it is easy to see that all three couplings approach a fixed point in the infrared. This fixed point can be explicitly calculated for the coupling (1.6). Employing a bare ghost-gluon vertex one obtains $\alpha^{g h-g l}(0) \approx 8.92 / N_{c}[11]$.

\section{Running coupling between Landau and Coulomb gauge}

One might expect that gauge invariant features of Yang-Mills theory show up in a quantity like the running coupling. Thus it is interesting to determine the coupling together with the infrared exponents of the corresponding dressing functions also in other gauges. A particularly interesting class of (transverse) gauges is specified by the gauge condition $a \partial_{0} A_{0}+\nabla \cdot \mathbf{A}=0$ with the gauge parameter $0 \leq a \leq 1$. The values $a=0,1$ correspond to Coulomb- and Landau-gauge respectively, whereas non-integer values of $a$ interpolate between these gauges.

In collaboration with D. Zwanziger we showed that in these gauges two renormalisation group invariant running couplings can be defined from the ghost-gluon vertex [5]. In the Landau gauge limit, $a=1$, these couplings unify to the expression (1.4). In the Coulomb gauge limit, however, they constitute two invariant charges which are given by

$$
\begin{aligned}
\alpha_{\text {coul }}(|\mathbf{k}|) & \equiv \frac{g^{2}}{4 \pi} Z_{00}^{\text {inst }}(|\mathbf{k}|), \\
\alpha_{I}(|\mathbf{k}|) & \equiv \frac{16}{3} \frac{g^{2}}{4 \pi} G^{2}(|\mathbf{k}|) \frac{Z_{\mathrm{et}}^{\mathrm{tr}}(|\mathbf{k}|)}{|\mathbf{k}|},
\end{aligned}
$$

where $Z_{00}^{\text {inst }}$ is the instantaneous part of the time-time component of the gluon dressing function and $Z_{\mathrm{et}}^{\mathrm{tr}}$ is the equal-time spatial gluon dressing function. The first coupling, $\alpha_{\text {coul }}$, is nothing else than the familiar colour Coulomb potential, which has been shown to be (almost) linearly rising $[3,13,14]$. The second coupling, $\alpha_{I}$, has a fixed point in the infrared [5]. This can be seen from the Dyson-Schwinger equation of the ghost propagator in Coulomb gauge. In interpolating gauges with $0<a \leq 1$, this fixed point can be calculated and we find the same value $\alpha_{I}(0) \approx 8.92 / N_{c}$ as in Landau gauge. Correspondingly, the ghost and gluon propagator obey an infrared power law with exponent $\kappa \approx 0.595$ independent of the gauge parameter $a$. The observation of these gauge independent features may have the potential to bring us closer to a unified picture of confinement in Landau and Coulomb gauge.

\section{Numerical solutions for the propagators in the continuum and on a torus}

There are at least three caveats in comparing results from the continuum Dyson-Schwinger approach to those of lattice calculations. First, the quantitative aspects of the continuum solutions 

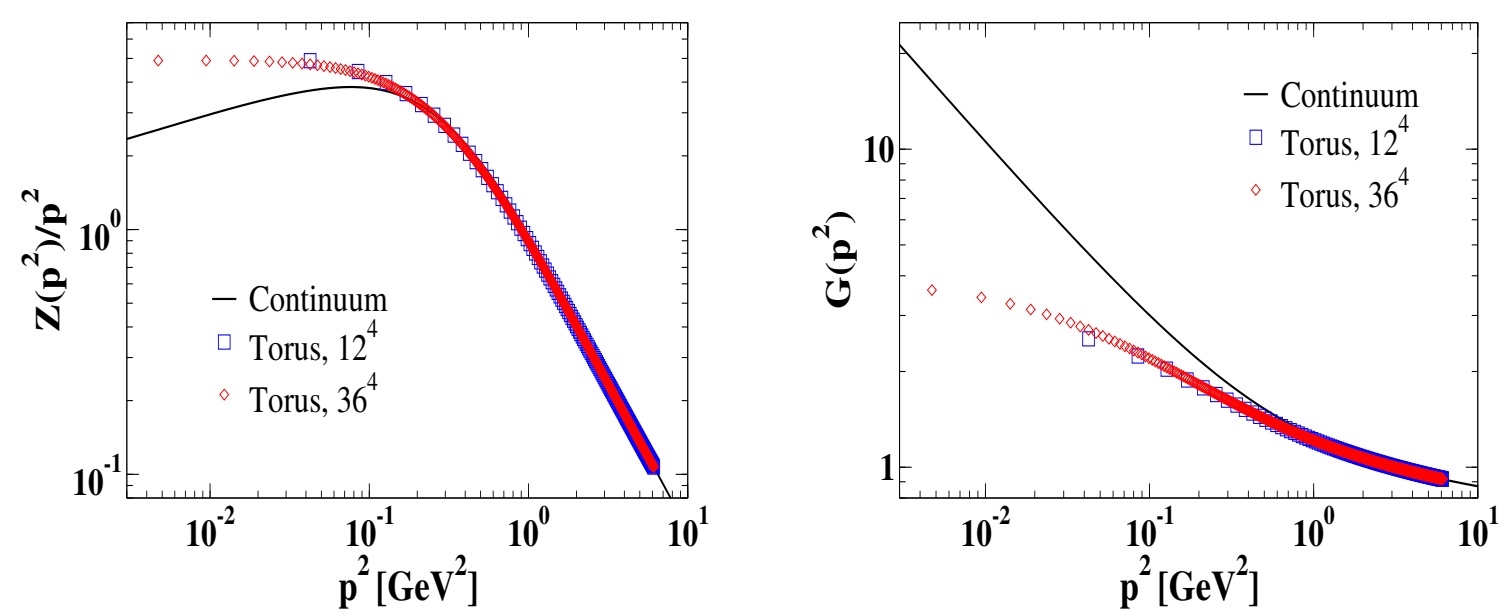

Figure 1: The gluon propagator function $Z\left(p^{2}\right) / p^{2}$ (left diagram) and the ghost dressing function $G\left(p^{2}\right)$ (right diagram). Compared are the results of the continuum DSEs with two respective solutions of DSEs on tori of different volumes.

depend on the details of the chosen truncation scheme, whereas the lattice calculations are ab initio. On the other hand, lattice calculations are carried out on a compact manifold, and therefore one has to deal with effects due to finite volume and lattice spacing. Finally, boundary conditions have to be chosen on the compact manifold. To quantify some of the latter two effects we formulated the Dyson-Schwinger equations on a torus without changing the truncation scheme $[6,15]$. One would then expect to see differences to the continuum solution for small volumes, which disappear continuously when the volume is chosen larger and larger. However, for our solutions this is not the case. In fig. 1 we compare solutions for the gluon propagator $Z\left(p^{2}\right) / p^{2}$ (left diagram) and the ghost dressing function $G\left(p^{2}\right)$ (right diagram) of the continuum with those from two different tori with volumes of $V \approx 2500 \mathrm{fm}^{4}$ and $V \approx 10^{5} \mathrm{fm}^{4}$. Note that the smaller volume corresponds to a typical one achieved in recent lattice simulations [16], whereas the larger value exceeds the current possibilities of the lattice formulation by far. Both solutions on the torus agree very well with each other, whereas there is a clear difference to the continuum results. In terms of the infrared exponent $\kappa$, defined in eq. (1.3), one finds a value of $\kappa \approx 0.5$ for the gluon propagator on the torus, which should be compared to the corresponding continuum value $\kappa \approx 0.6$. Similarly, whereas the ghost dressing function diverges in the continuum, we observe a finite ghost on the compact manifold. These differences persist, when the infrared behaviour on the torus is extrapolated to the continuum $[6]$.

We compare these results to the ones of recent lattice simulations in fig. 2 and fig. 3. The lattice simulations for the gluon propagator are all performed for $\mathrm{SU}(3)$ at different values of $\beta$, different lattice sizes and different actions $[16,17,18]$ (see also [19]). They fall on top of each other. On the other hand there are slight differences between the SU(2) ghost dressing function of ref. [20] and the SU(3) result of ref. [18] (see also [21]). Interestingly, there is nice qualitative agreement between both, the lattice and the DSE-formulation on the compact manifold. Both approaches give a finite gluon propagator in the infrared. This is in marked difference to the numerical continuum result, which agrees with the analytic power law, eq.(1.3), with $\kappa \approx 0.6$. The situation is less clear for the ghost dressing function, though at least the SU(3) result of ref. [18] seems to follow more 

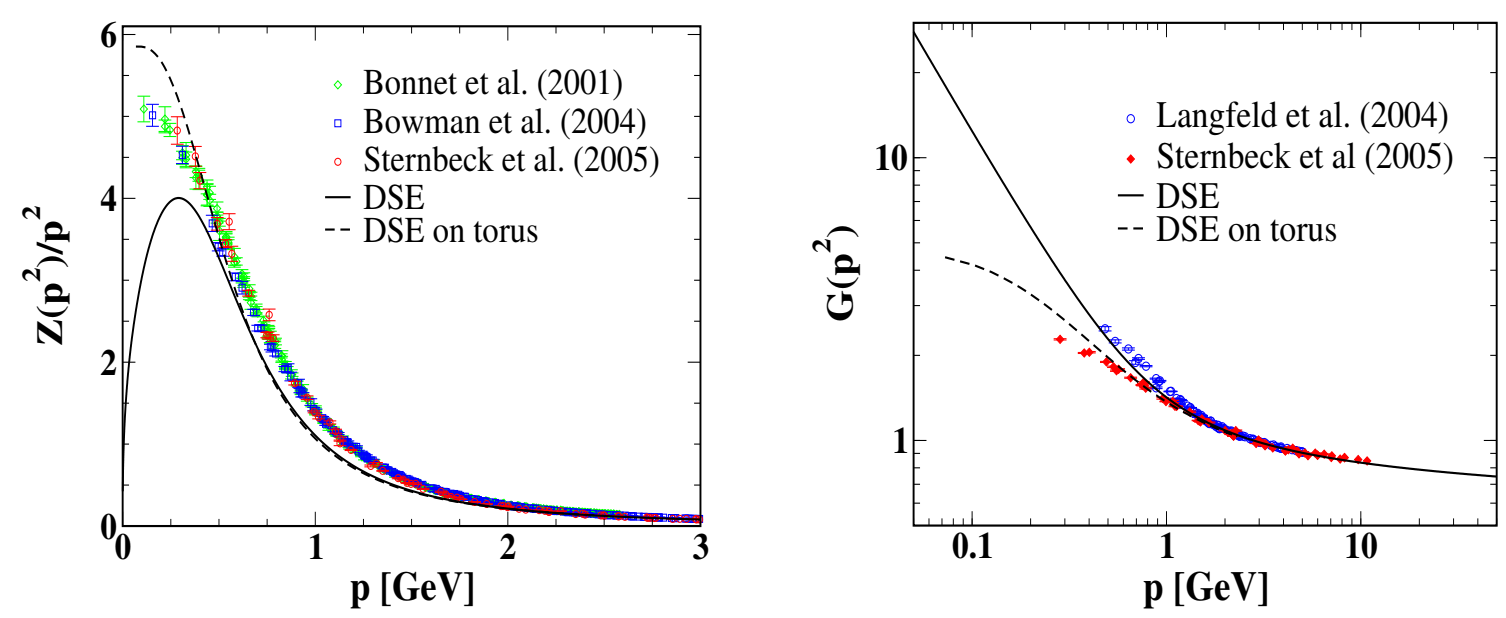

Figure 2: The results for gluon and ghost from Dyson-Schwinger equations in the continuum and on the torus are compared with the lattice data of refs. $[16,17,18,20]$

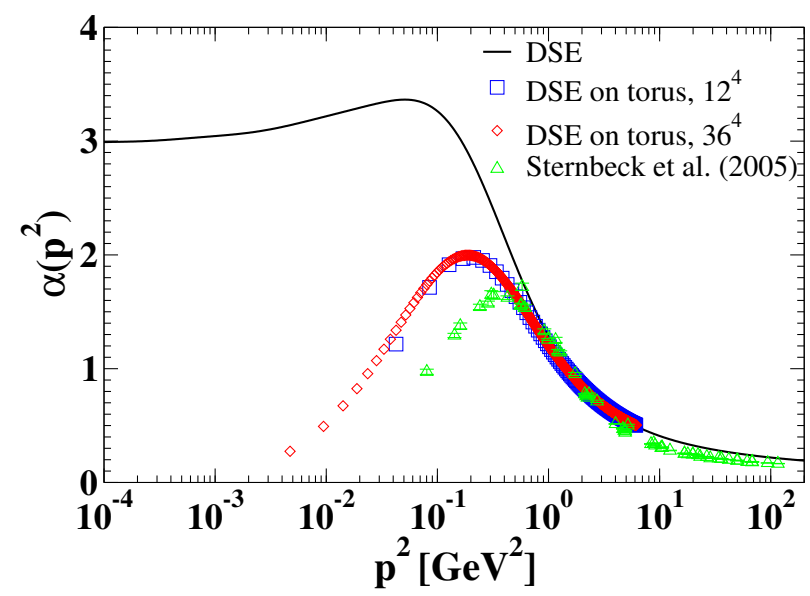

Figure 3: Results for the running coupling of the ghost-gluon vertex from DSEs in the continuum and on the torus compared to the lattice data of ref. [18].

or less the DSE-solution on the torus. For the running coupling $\alpha^{g h-g l}\left(p^{2}\right)=\frac{g^{2}}{4 \pi} G^{2}\left(p^{2}\right) Z\left(p^{2}\right)$ the numerical solution in the continuum reproduces the fixed point $\alpha^{g h-g l}(0) \approx 8.92 / N_{c}$ discussed below eq.(1.6). On the compact manifold, however, the coupling vanishes at small momenta ${ }^{1}$, a behaviour which seems to be at least counterintuitive.

Our results from DSEs compared to lattice simulations of the ghost and gluon propagators show an interesting difference between the infrared behaviour found in the continuum and the one found on a compact manifold. Finite volume effects can hardly explain this result, since the difference persists for extremely large tori. The role of different boundary conditions in this respect is currently investigated (c.f. also ref. [23], these proceedings).

\footnotetext{
${ }^{1} \mathrm{~A}$ similar result has been found for the running coupling of the three-gluon vertex in ref. [22]. Note, however, that the conclusions drawn there are contradicted by the results of refs. [4, 7, 8, 11].
} 


\section{Acknowledgements}

I would like to thank the organisers of Lattice 2005 for all their efforts which made this interesting conference possible. I am grateful to R. Alkofer, B. Gruter, F. Llanes-Estrada and D. Zwanziger for fruitful collaboration on the topics presented here. I thank D. Leinweber, O. Oliveira, M. Pennington, J. Skullerud and A. Williams for inspiring discussions. This work has been supported by the Deutsche Forschungsgemeinschaft (DFG) under contract Fi 970/2-1.

\section{References}

[1] N. Nakanishi and I. Ojima, World Sci. Lect. Notes Phys. 27, 1 (1990).

[2] R. Alkofer and L. von Smekal, Phys. Rept. 353, 281 (2001).

[3] D. Zwanziger, Phys. Rev. D 70 (2004) 094034.

[4] R. Alkofer, C. S. Fischer and F. J. Llanes-Estrada, Phys. Lett. B 611, 279 (2005).

[5] C. S. Fischer and D. Zwanziger, Phys. Rev. D 72 (2005) 054005.

[6] C. S. Fischer, B. Gruter and R. Alkofer, arXiv:hep-ph/0506053.

[7] P. Watson and R. Alkofer, Phys. Rev. Lett. 86 (2001) 5239.

[8] A. Cucchieri, T. Mendes and A. Mihara, JHEP 0412 (2004) 012.

[9] W. Schleifenbaum, A. Maas, J. Wambach and R. Alkofer, Phys. Rev. D 72 (2005) 014017.

[10] D. Zwanziger, Phys. Rev. D 65, 094039 (2002).

[11] C. Lerche and L. von Smekal, Phys. Rev. D 65, 125006 (2002).

[12] J. M. Pawlowski, D. F. Litim, S. Nedelko and L. von Smekal, Phys. Rev. Lett. 93 (2004) 152002 ; C. S. Fischer and H. Gies, JHEP 0410 (2004) 048,

[13] J. Greensite and S. Olejnik, Phys. Rev. D 67 (2003) 094503.

[14] C. Feuchter and H. Reinhardt, Phys. Rev. D 70 (2004) 105021; C. Feuchter and H. Reinhardt, arXiv:hep-th/0402106.

[15] C. S. Fischer and R. Alkofer, Phys. Lett. B 536, 177 (2002);

C. S. Fischer, R. Alkofer and H. Reinhardt, Phys. Rev. D 65, 094008 (2002).

[16] F. D. Bonnet et al., Phys. Rev. D 64, 034501 (2001).

[17] P. O. Bowman, U. M. Heller, D. B. Leinweber, M. B. Parappilly and A. G. Williams, Phys. Rev. D 70 (2004) 034509.

[18] A. Sternbeck, E. M. Ilgenfritz, M. Mueller-Preussker and A. Schiller, Phys. Rev. D 72 (2005) 014507.

[19] O. Oliveira and P. J. Silva, AIP Conf. Proc. 756, 290 (2005).

[20] J. Gattnar, K. Langfeld and H. Reinhardt, Phys. Rev. Lett. 93, 061601 (2004).

[21] S. Furui and H. Nakajima, Phys. Rev. D 69 (2004) 074505.

[22] P. Boucaud et al., JHEP 0304, 005 (2003); P. Boucaud, F. De Soto, A. Le Yaouanc, J. P. Leroy, J. Micheli, O. Pene and J. Rodriguez-Quintero, arXiv:hep-ph/0505150.

[23] T. Tok, L. v. Smekal, K. Langfeld, H. Reinhardt, "The gluon propagator in lattice Landau gauge with twisted boundary conditions,",these proceedings. 